\title{
Sex Differences of Clinical Presentation and Outcomes in Propensity-Matched Patients with Acute Type A Aortic Dissection
}

\author{
Yang Li, MD*, ${ }^{1}$ Nan Yang, MD*, ${ }^{2}$ Siqi Liu, MD,${ }^{3}$ Weixun Duan, MD,${ }^{4}$ Min Zeng, MD,${ }^{5}$ Dinghua Yi, MD \\ ${ }^{1}$ Department of Cardiac Surgery, Xuanwu Hospital, Capital Medical University, Beijing, China; \\ ${ }^{2}$ Department of Stomatology, The 8th Medical Center of PLA, Beijing, China; \\ ${ }^{3}$ Department of Cardiovascular Surgery, First Affiliated Hospital, Harbin Medical University, Harbin, China; \\ ${ }^{4}$ Department of Cardiovascular Surgery, Xijing Hospital, Air Force Medical University, Xi'an, China; \\ ${ }^{5}$ Department of Internal Medicine, Beijing Yuho Rehabilitation Hospital of Integrated Traditional Chinese and Western Medicine, \\ Beijing, China
}

\section{ABSTRACT}

Objectives: To assess sex differences of clinical presentation and outcomes in propensity-matched patients with acute type A aortic dissection (AAAD).

Methods: We collected the clinical data of patients with AAAD from a single heart center between January 2009 and July 2014. After propensity score matching, we compared differences in clinical presentation and outcomes of patients with AAAD between men and women.

Results: There were 590 patients $(295$ men and 295 women) with AAAD through propensity matching on demographics and patients' history. We found that the presentation and diagnosis of AAAD often were more delayed in women. Severe signs of congestive heart failure $(9.8 \%$ vs. $5.1 \%, P=$ $0.017)$, cardiac tamponade/shock $(9.1 \%$ vs. $4.1 \%, P<0.001)$, and periaortic hematoma $(26.4 \%$ vs. $21.7 \%, P<0.001)$ were more commonly presented in women. Surgery was more commonly performed in men than in women $(95.4 \%(281 / 295)$ vs. $91.5 \%(270 / 295), P=0.045)$, indicating the association of sex with surgical decision. To investigate the association of sex with outcomes after surgery, patients who underwent surgical treatment were re-matched (262 men and 262 women) by propensity score. Women suffered from greater in-hospital mortality than men $(8.4 \%$ vs. $3.4 \%, P<0.001)$. Postoperative complications of congestive heart failure $(9.1 \%$ vs. $3.8 \%, P<$ $0.001)$, visceral ischemia (6.8\% vs. $1.1 \%, P<0.001)$, and limb ischemia $(7.6 \%$ vs. $1.5 \%, P<0.001)$ were more frequent in women. For women, prolonged operative time may increase in-hospital mortality, especially after 12 hours from the start of surgery $(30.0 \%$ vs. $14.3 \%, P<0.001)$. Kaplan-Meier survival analysis indicated worse late outcomes in women in the matched surgery group $(\log$-rank $P=0.012)$.

Conclusions: Our analysis provides new insights into sex differences in clinical presentation and outcomes of AAAD.

Received fanuary 15, 2021; accepted Fanuary 27, 2021

*Dr. Yand Li and Dr. Nan Yang contributed equally to this article.

Correspondence: Dinghua Yi, MD, No 15, Changle West Road, Xi'an, China, Telephone or Fax +86-029-84775507 (e-mail: heart055@sohu.com)

\section{INTRODUCTION}

The role of sex in influencing the outcome of adverse health events is an area of considerable interest and importance. Emerging evidence shows that sex differences in cardiovascular disease incidence and outcomes need to be recognized and incorporated in the effective and optimal management and treatment of disease. Acute type A aortic dissection (AAAD) is a catastrophic cardiovascular disease with a high mortality. Untreated mortality of AAAD has been reported to be approximately $1 \%$ to $2 \%$ per hour after symptom onset, with up to $90 \%$ of patients succumbing within two weeks. However, very few studies have looked at the sex difference of AAAD [Rawla 2019; Pawlukiewicz 2020]. Although the International Registry of Acute Aortic Dissection (IRAD) reported sex-related differences in acute aortic dissection, these data did not necessarily reflect the status of the general population due to different races, medical insurance, and socio-cultural backgrounds [Evangelista 2016; Tsai 2009; Berretta 2016].

Propensity score matching frequently is used to estimate the effect of treatments, exposures, and interventions when using observational data. This method will advocate the position that observational studies can and should be designed to approximate randomized experiments as closely as possible. Therefore, the aim of the present study was to evaluate sex differences in clinical presentation and outcomes in a large group of propensity-score matched patients with AAAD. We anticipated that this analysis would provide helpful information distinguishing clinical features and improving management and outcomes between men and women.

\section{METHODS}

Data collection: Clinical data of patients with AAAD from the Department of Cardiovascular Surgery at Xijing Hospital of Air Force Medical University were obtained between January 2009 and July 2014 through retrospective chart review by trained cardiovascular researchers. The data collection form included 140 variables with demographic information, medical record, physical examination, imaging techniques, treatment, in-hospital complications, and mortality. Diagnosis was 
based on history and physical examination and confirmed by imaging, visualization at surgery, and/or postmortem examination. All patients were followed up for six years after discharge by clinic chart review or telephone interview.

Creating matched cohorts: To design observational data to an approximate random study as closely as possible, male and female patients were matched on the propensity score. We first matched on demographics and patients' history for beneficiaries to compare differences in clinical presentation between men and women. To investigate the association of sex with outcomes after surgery, patients who underwent surgical treatment were re-matched on surgical variables. Each beneficiary was matched with the closest estimated propensity score. To ensure close matches, we required that the estimated log-odds scores for male and female patients be within 0.20 standardized difference (SD). This requirement ensures the removal of approximately $90 \%$ of the bias, due to differences in covariate distributions between men and women.

The rationale for this study previously has been described [Zhang 2017; Sun 2011]. The study protocol was approved by the institutional review board of Army General Hospital and waived the need for written consent.

Definitions: AAAD was defined as any dissection that involved the ascending aorta within 14 days of the onset of symptoms. Intramural hematoma was defined as a dissecting membrane without any degree of patency of the false lumen. Coma was defined as complete mental unresponsiveness without psychologically or physiologically appropriate responses to stimulation. Spinal cord ischemia was defined as loss of function of the extremities with or without bowel/bladder dysfunction due to occlusion of radicular spinal cord arteries. Stroke was considered a loss of neurologic function due to an acute ischemic injury to the brain with residual symptoms. Cardiac tamponade/shock was defined as a cardiogenic shock with a systolic blood pressure of $90 \mathrm{mmHg}$, associated with pericardial effusion confirmed by computed tomography (CT) or echocardiography. Hospital mortality included death during the hospitalization and death within 30 days of the operation. Late mortality included all deaths during the available follow-up period.

Operative techniques: Surgery was performed by median sternotomy and cardiopulmonary bypass (CPB). Cannulation of the femoral artery was performed in patients with only ascending aortic involvement. In patients with hemiarch replacement and total arch replacement (TAR), cannulation of the right axillary artery was used for CPB and selective cerebral perfusion. In patients requiring hemiarch replacement, the transverse aortic arch was opened and inspected closely. The lesser curvature of the aortic arch was resected. A beveled incision was made for the distal end of a vascular graft to replace the arch. After completion of the open distal anastomosis, the graft was cross-clamped, and antegrade systemic perfusion resumed through a side branch. The method of TAR combined with a stented elephant trunk has been described in previous detail [Zhong 2018; Fukui 2015; Pape 2015]. An intraoperative stent-graft (MicroPort Medical Company Limited, Shanghai, China) and a four-branched prosthetic graft (Boston Scientific Inc, Boston, MA) were employed in this implantation. The distal aorta was transected circumferentially between the origin of the left common carotid artery and the origin of the left subclavian artery. The stent was implanted into the distal aorta. The distal aorta, incorporating the stented elephant trunk, was firmly attached to the distal end of the four-branched prosthetic graft using the "open" aortic method. Antegrade systemic perfusion was reestablished through the perfusion limb of the four-branched prosthetic graft. The anastomosis to the left common carotid artery was carried out first. After the anastomosis was completed, $\mathrm{CPB}$ was gradually returned to normal flow, selective cerebral perfusion was discontinued, and rewarming was started. The anastomosis to the left subclavian artery, the innominate artery, and the proximal anastomosis were completed.

Statistical analysis: Data of collected patients are presented as frequencies and percentages for categorical variables, and as mean $\pm \mathrm{SD}$ for continuous variables. In all cases, missing data were not defaulted to negative, and denominators reflect only cases reported. We used Fisher's test for categorical variables and unpaired $t$ tests for continuous variables. Moreover, we created a propensity score-matched cohort (a 1:1 match) by using a greedy matching technique. After matching, crude comparisons of the matched cohorts were made using the McNemar's test and paired t tests. Multivariable logistic regression was used to analyze risk factors for death before surgery. Survival rates were estimated with the use of Kaplan-Meier methods and comparisons were made with the use of log-rank analysis. A $P$ value $<0.05$ was considered statistically significant.

\section{RESULTS}

Clinical presentation after propensity matching: In the original sample, the data of 753 patients with AAAD (451 men and 302 women) were collected. There were some differences in basic characteristics of demographics and patients' history between the sexes. Median age was 42 (range: 18 to 85 years) and 50 (range: 19 to 83 years) years in men and women, respectively. Compared with men, women were significantly older in the mean age $(50.7 \pm 12.3$ years vs. $41.2 \pm 11.3$ years, $P$ $<0.001)$. Men were more likely to have a history of hypertension $(85.7 \%$ vs. $79.8 \%, P<0.001)$ and smoking $(53.9 \%$ vs. $12.9 \%, P<0.001)$ compared with women.

To compare differences in clinical presentation between men and women under equal conditions, patients were propensity-score matched on basic characteristics of demographics and patients' history. There were 590 patients with 295 in each cohort. After matching, we found some differences in clinical presentation, signs and imaging findings between men and women. (Table 1) The interval from symptom onset to initial hospitalization revealed that women had more often presented later than men. Moreover, time from symptom onset to diagnosis also was delayed in women. Although $22.7 \%$ of men and $19.3 \%$ of women were diagnosed early (within 4 hours), relatively more women $(44.7 \%)$ were diagnosed after 24 hours of symptom onset. Typical chest with abrupt onset was similar between men and women. Compared 
Table 1. Comparison of clinical presentation and imaging findings between men and women in the matched sample

\begin{tabular}{|c|c|c|c|c|}
\hline \multicolumn{5}{|l|}{ Clinical presentation } \\
\hline Time from symptom onset to diagnosis ( $h$ ) & $34.8 \pm 12.9$ & $43.2 \pm 16.5$ & 0.268 & $<0.001$ \\
\hline$<4 \mathrm{~h}(\%)$ & $67(22.7)$ & $57(19.3)$ & 0.021 & 0.304 \\
\hline$>24 \mathrm{~h}(\%)$ & $115(38.9)$ & $132(44.7)$ & 0.593 & $<0.001$ \\
\hline Chest pain (\%) & $215(72.9)$ & $218(73.9)$ & 0.115 & 0.218 \\
\hline Back pain (\%) & $94(31.9)$ & $87(31.8)$ & 0.291 & 0.075 \\
\hline Migrating pain (\%) & $76(25.8)$ & $69(23.4)$ & 0.069 & 0.104 \\
\hline Syncope (\%) & $10(3.4)$ & $13(4.4)$ & 0.135 & 0.066 \\
\hline Limb ischemia (\%) & $6(2.0)$ & $10(3.4)$ & 0.051 & 0.462 \\
\hline \multicolumn{5}{|l|}{ Diagnostic imaging findings } \\
\hline Aortic regurgitation requiring valve replacement (\%) & $79(26.8)$ & $82(27.8)$ & 0.131 & 0.234 \\
\hline Coronary artery compromise (\%) & $59(20.0)$ & $65(22.0)$ & 0.154 & 0.079 \\
\hline Periaortic hematoma (\%) & $64(21.7)$ & $78(26.4)$ & 0.327 & $<0.001$ \\
\hline Arch vessel involvement (\%) & $95(32.2)$ & $89(30.2)$ & 0.259 & 0.127 \\
\hline Intramural hematoma (\%) & $28(9.5)$ & $24(14.9)$ & 0.336 & 0.342 \\
\hline Pericardial effusion (\%) & $59(20.0)$ & $70(23.7)$ & 0.144 & 0.136 \\
\hline Pleural effusion (\%) & $48(16.3)$ & $55(18.6)$ & 0.743 & 0.091 \\
\hline
\end{tabular}

Data were presented as mean \pm standardized deviation or percentage. SD, standardized difference

with men, women were more likely to present with congestive heart failure, cardiac tamponade/shock. Imaging sign of periaortic hematoma was more commonly seen in women.

Association of sex with mortality before surgery: In the matched sample, 25 women and 14 men were dead before surgery. The mortality was similar between men and women (8.5\% vs. $4.7 \%, P=0.068)$. Multivariable logistic analysis indicated that aortic rupture (95\% CI 1.62-3.84, OR 1.65, $P$ $=0.036)$, acute myocardial infarction $(95 \%$ CI 1.31-2.95, OR $1.27, P=0.019)$, and congestive heart failure (95\% CI 1.03 2.46 , OR $1.08, P=0.017$ ) were important factors for death before surgery.

Association of sex with outcomes after surgery: Surgery was more commonly performed in men than in women (95.4\% (281/295) vs. $91.5 \%(270 / 295), P=0.045)$, indicating the association of sex with surgical decision. To investigate association of sex with outcomes after surgery, patients who underwent surgical treatment were re-matched on the propensity score. After matching, there were 524 patients with 262 in each group. (Table 2)

In the matched surgery group, women suffered from greater mortality than men $(8.4 \%(22 / 262)$ vs. $3.4 \%(9 / 262), P<$

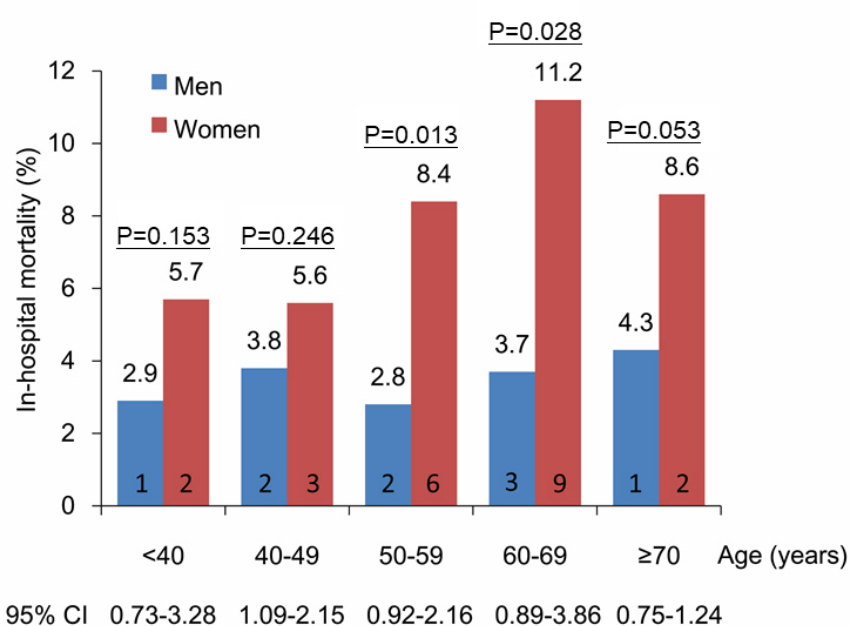

Figure 1. In-hospital mortality for both genders with AAAD in different age groups. Absolute numbers of patients were shown at the bottom of bars and $P$ value at the top of bars. Confidence limits of each group were listed at the bottom of each column. 
Table 2. Comparison of surgical data between men and women in the rematched sample

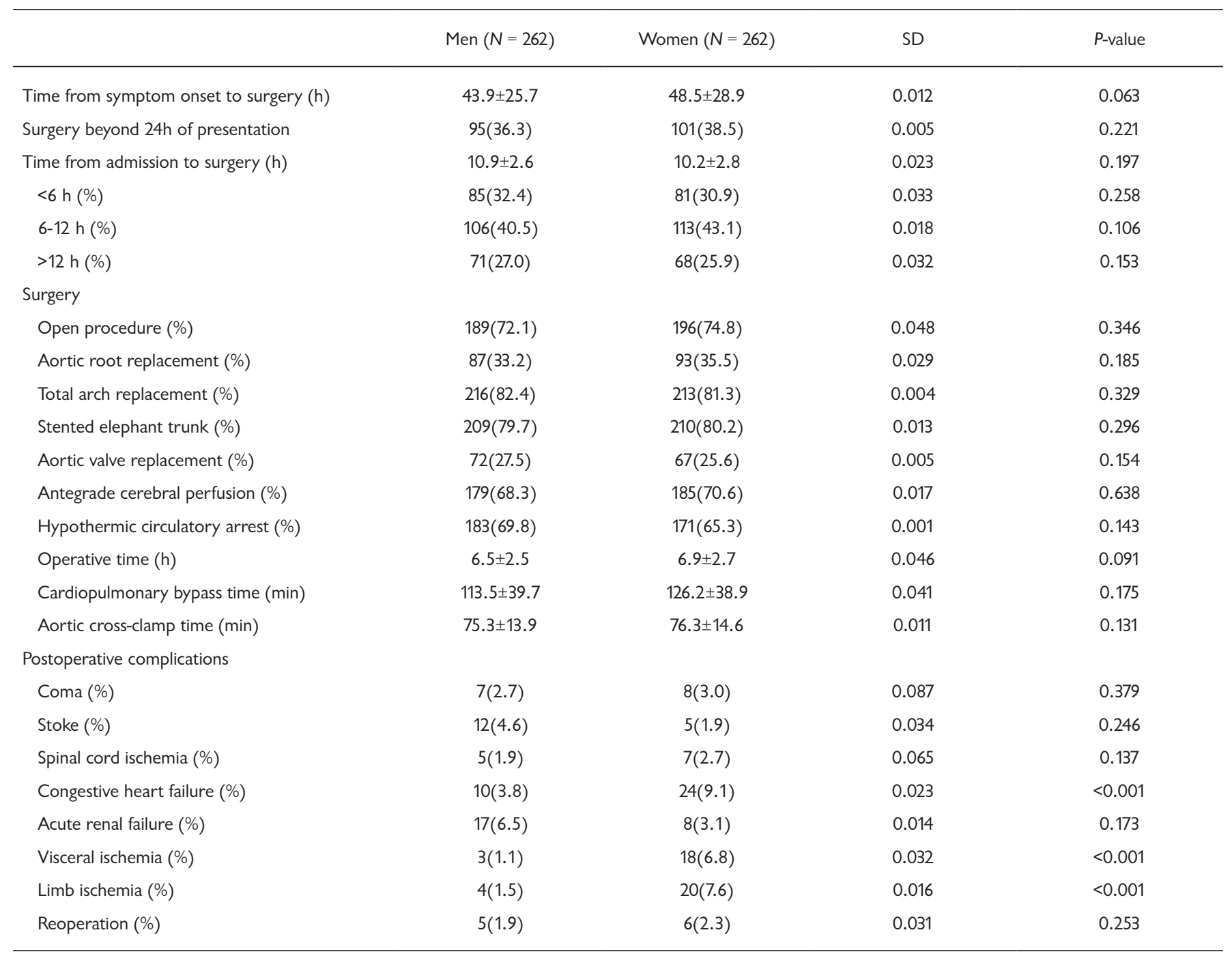

Data were presented as mean \pm standardized deviation or percentage. SD, standardized difference

0.001). Considering different age groups, major differences in hospital mortality between women and men were presented in both age groups of 50 to 59 and 60 to 69 years, whereas other age groups showed no differences in gender-specific mortality. (Figure 1) Moreover, prolonged operative time may increase in-hospital mortality, particularly in women. (Figure 2) After 12 hours from the start of surgery, in-hospital mortality was significantly higher in women than in men. In-hospital complications of congestive heart failure, visceral ischemia and limb ischemia were more likely to present in women.

Follow up: In the follow up, 26 men and 50 women died from aortic rupture, ischemic cardiac disease, cerebrovascular disease, cardiac valve disease, pulmonary diseases, septicemia, and other unrelated causes of death. The causes of death were no different between men and women. Kaplan-Meier survival curves stratified by sex indicated worse late outcomes in women in the matched surgery group. (Figure 3)

\section{DISCUSSION}

Current study provides new insight into sex-related differences in clinical presentation and outcomes in a large group of matched patients with AAAD. We found that men differed from women in several important aspects. Several of these differences have important diagnosis and treatment implications in female patients with AAAD.

In the first matched sample (295 men and 295 women), some important differences were found in clinical presentation between men and women (Table 2). Time interval from insult to diagnosis is an important parameter that affects AAAD outcomes, and our study showed some interesting data in this regard. Specifically, there was a 8.4-hour delay in women compared with men for interval from symptom onset to diagnosis $(P<0.001)$. In addition, a significantly greater percent of women was diagnosed after $>24$ hours, 
after symptom onset compared with men (44.7\% vs. $38.9 \%$, $P<0.001)$, indicating that diagnosis of AAAD was made later in women than men on average. This finding was consistent with the results from the IRAD. However, there was a significantly greater delay in diagnosis of AAAD in our study as compared with the IRAD [Zhong 2018; Fukui 2015; Pape 2015]. This is because women were less likely to experience the typical acute onset of chest pain. Congestive heart failure and cardiac tamponade/shock also were more common presentation in women than in men. These severe manifestations often are associated with heart diseases and may cause a neglected diagnosis of acute aortic dissection in women. Given the potentially catastrophic results of delayed detection, physicians must maintain comprehensive thinking for AAAD diagnosis in female patients.

AAAD needs urgent surgery after diagnosis. In the first matched samples, more than $90 \%$ of patients underwent surgical treatment. This result suggested that we used a more aggressive approach to treat AAAD compared with IRAD [Wang 2014; Tamura 2019; Moeller 2019]. Actually, an aggressive and early surgical treatment is the only option to provide a reasonable chance of survival and prevent many complications. However, there is no standard procedure for surgical treatment of AAAD. Whether limited replacement of the ascending aorta with resection of the intimal tear or TAR should be carried out in patients with AAAD is controversial [Cekmecelioğlu 2020]. IRAD reported that the majority of patients $(81.7 \%$ ) with AAAD underwent ascending aortic replacement with resection of the intimal tear, and fewer patients $(10.6 \%)$ were treated by TAR [Sun 2011]. In fact, an ideal therapy should be able to replace the dissected aortic segment as long as possible and obliterate the false lumen, which is essential to minimizing operative risks and avoiding or reducing the need for reinterventions. Dr. Sun designed a stented graft in 2002 and proposed a modified technique of TAR using a four-branched graft with implantation of stented elephant trunk (Sun's procedure) as a treatment for AAAD [Li 2020]. This modified technique of TAR was employed in approximately $80 \%$ of patients with AAAD in our study. Sun's procedure can simplify operative method and decrease operative time and postoperative complications. At present, nearly 8000 patients with AAAD in China have received TAR using Sun's procedure and produced satisfactory outcomes [Divchev 2015].

In an attempt to investigate outcomes after surgery, patients who underwent surgical repair were re-matched on the propensity score. After the second matching, we found that women had worse early outcomes. In-hospital mortality may be related to operative time. Using time of surgery as a time-varying covariable, we investigated in-hospital mortality between both genders in different operative time. Our results further substantiated that prolonged operative time may increase in-hospital mortality, especially in women. Thus, shortening operative time could be an approach to reduce in-hospital mortality. Moreover, in-hospital morbidity data showed that there was a significant increase in the occurrence of congestive heart failure, visceral ischemia, and limb ischemia in women compared with men. A higher percent of

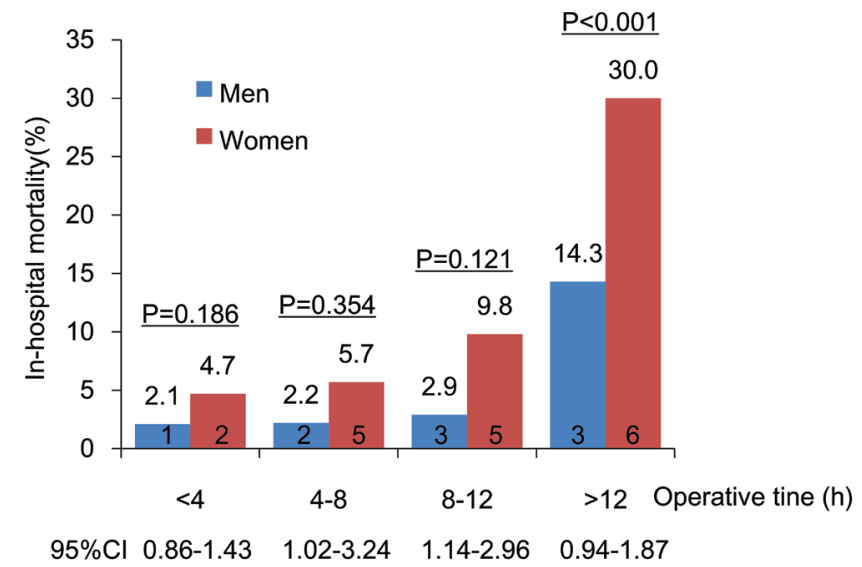

Figure 2. In-hospital mortality for both genders with AAAD in different operative time. Absolute numbers of patients were shown at the bottom of bars and $P$ value at the top of bars. Confidence limits of each group were listed at the bottom of each column.

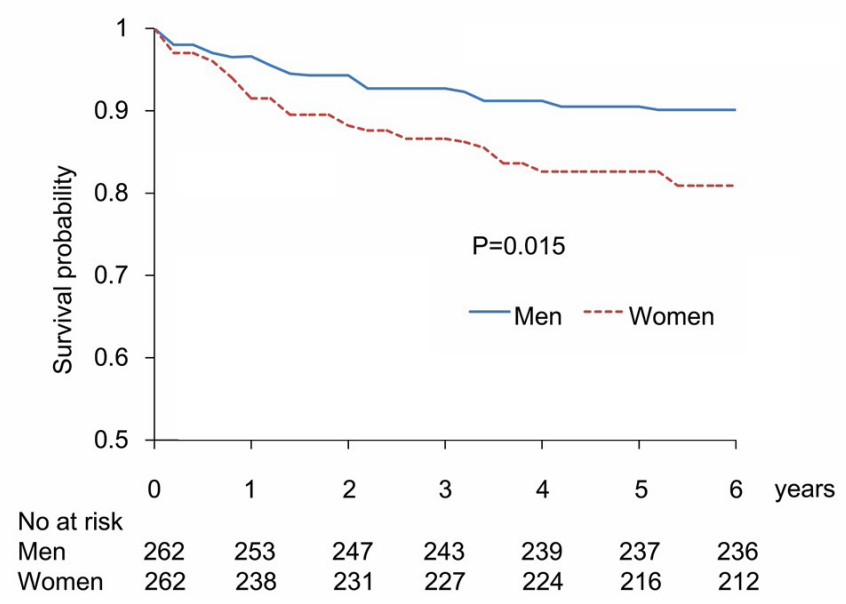

Figure 3. Kaplan-Meier survival curves stratified by sex in patients treated surgically in the matched surgery group. Confidence limits of each period were listed at the bottom of each column.

in-hospital morbidity may be a main cause of severe outcomes. Interestingly, our study had a lower morbidity and mortality compared with the IRAD and other studies [Berretta 2016]. The differential result may be associated with surgical technique, operative time, or hemodynamics at surgery.

Our study also investigated long-term outcomes of AAAD in the propensity-score-matched surgery group. The result showed that women, who underwent surgical treatment, had worse late outcomes in the follow up. Previous study indicated that higher late mortality in women treated surgically was associated with outcomes under high-risk condition before operation. In our study, selective bias was excluded as much as possible to compare differences in outcome between men and women under equivalent condition. In the rematched surgery group, we had no significant variation in surgical technique, delay, or hemodynamics at surgery between genders, the 
differential late outcomes of women appear to be both real and possibly explained by inherently higher risk at surgery. Studies suggested that female gender was considered as an independent predictor for the mortality of patients with cardiovascular diseases [Smedberg 2020]. By Kaplan-Meier analysis, our result also supported a concept that female gender had worse late outcomes after repair of AAAD.

There are some limitations. First, we represented the experience of a single center. These data do not represent outcomes of the overall population. Second, nonfatal adverse events, functional status, and resource use also must be taken into consideration. Finally, risk factors which affected early or late death need to be evaluated in future studies.

In summary, our study evaluated sex-related differences in clinical presentation and outcomes of AAAD in the propensity-score matched sample. These differences had very important implications for improving diagnosis and treatment and should be paid more attention by clinical physicians. The development of strategy to identify and treat AAAD may improve clinical outcomes, especially in female patients with AAAD.

\section{ACKNOWLEDGEMENT}

We would like to thank the Department of Cardiovascular Surgery, Xijing Hospital, Air Force Medical University. This study was supported by the College-level Project in Xuanwu Hospital (No: XWJL-2019024) and the National Natural Science Foundation (No: 81400854).

\section{REFERENCES}

Berretta P, Patel HJ, Gleason TG, Sundt TM, Myrmel T, Desai N, et al. 2016. IRAD experience on surgical type A acute dissection patients: results and predictors of mortality[J]. Ann Cardiothorac Surg. 5(4): 346-351.

Çekmecelioğlu D, Köksoy C, Coselli J. 2020. The frozen elephant trunk technique in acute DeBakey type I aortic dissection[J]. Turk Gogus Kalp Damar Cerrahisi Derg. 28(3): 411-418.

Divchev D, Najjar T, Tillwich F, Rehders T, Palisch H, Nienaber CA. 2015. Predicting long-term outcomes of acute aortic dissection: a focus on gender. Expert Rev Cardiovasc Ther. 13:325-31.

Erbel R, Aboyans V, Boileau C, Bossone E, Bartolomeo RD, Eggebrecht H, et al. 2014. 2014 ESC Guidelines on the diagnosis and treatment of aortic diseases: Document covering acute and chronic aortic diseases of the thoracic and abdominal aorta of the adult. The Task Force for the Diagnosis and Treatment of Aortic Diseases of the European Society of Cardiology (ESC). Eur Heart J. 35:2873-926.
Evangelista A, Maldonado G, Gruosso D, Teixido G, Rodríguez-Palomares J, Eagle K. 2016. Insights from the International Registry of Acute Aortic Dissection[J]. Glob Cardiol Sci Pract. 2016(1): e201608.

Fukui T, Tabata M, Morita S, Takanashi S. 2015. Gender differences in patients undergoing surgery for acute type A aortic dissection. J Thorac Cardiovasc Surg. 150:581-587.

Li X, Qiao H, Shi Y, Xue J, Bai T, Liu Y, Sun L. 2020. Role of proximal and distal tear size ratio in hemodynamic change of acute type A aortic dissection. J Thorac Dis. 12(6): 3200-3210.

Moeller E, Nores M, Stamou SC. 2019. Repair of Acute Type-A Aortic Dissection in the Present Era: Outcomes and Controversies. Aorta (Stamford). 7(6): 155-162.

Pape LA, Awais M, Woznicki EM, Suzuki T, Trimarchi S, Evangelista A, et al. 2015. Presentation, Diagnosis, and Outcomes of Acute Aortic Dissection: 17-Year Trends From the International Registry of Acute Aortic Dissection. J Am Coll Cardiol. 66:350-8.

Pawlukiewicz AJ, Long D, Mehta S. 2020. Large Vessel Occlusion Stroke Secondary to Acute Aortic Dissection. Cureus. 12(7): e9278.

Rawla P, El Helou ML, Vellipuram AR. 2019. Fluoroquinolones and the Risk of Aortic Aneurysm or Aortic Dissection: A Systematic Review and Meta-Analysis[J]. Cardiovasc Hematol Agents Med Chem. 17(1): 3-10.

Smedberg C, Steuer J, Leander K, Hultgren R. 2020. Sex differences and temporal trends in aortic dissection: a population-based study of incidence, treatment strategies, and outcome in Swedish patients during 15 years. Eur Heart J. 41(26): 2430-2438.

Sun L, Qi R, Zhu J, Liu Y, Chang Q, Zheng J. 2011. Repair of acute type A dissection: our experiences and results. Ann Thorac Surg. 91(4):1147-52.

Sun L, Qi R, Zhu J, Liu Y, Zheng J. 2011. Total arch replacement combined with stented elephant trunk implantation: a new "standard" therapy for type a dissection involving repair of the aortic arch? Circulation. 123:971-978.

Tamura K, Chikazawa G, Hiraoka A, Totsugawa T, Yoshitaka H. 2019. Characteristics and Surgical Results of Acute Type A Aortic Dissection in Patients Younger Than 50 Years of Age. Ann Vasc Dis. 12(4): 507-513.

Tsai TT, Trimarchi S, Nienaber CA. 2009. Acute aortic dissection: perspectives from the International Registry of Acute Aortic Dissection (IRAD). Eur J Vasc Endovasc Surg. 37:149-159.

Wang W, Duan W, Xue Y, Wang L, Liu J, Yu S, et al. 2014. Clinical features of acute aortic dissection from the Registry of Aortic Dissection in China. J Thorac Cardiovasc Surg. 148:2995-3000.

Zhang Q, Ma X, Zhang W, Wang Z, Zhang H, Zhang X, et al. 2017. Surgical repair and reconstruction of aortic arch in debakey type I aortic dissection: recent advances and single-center experience in the application of branched stent graft. J Cardiothorac Surg. 12: 86.

Zhong YL, Qi RD, Ma WG, Ge YP, Qiao ZY, Li CN, et al. 2018. Frozen elephant trunk with modified en bloc arch reconstruction and left subclavian transposition for chronic type A dissection. J Thorac Dis. 10(9): 5376-5383. 